Institut der Musikwissenschaft, Philosophische Fakultät, Karlsuniversität, Prag, Abteilung der Komposition, Musikfakultät, Akademie der musischen Künste, Prag Muzikološki inštitut, Filozofska fakulteta, Karlova univerza, Praga

Oddelek za kompozicijo, Glasbena fakulteta, Akademija glasbenih umetnosti, Praga

\title{
Tschechische musikalische Identität in mitteleuropäischem Kontext am Anfang des 21. Jahrhunderts
}

\author{
Češka glasbena identiteta \\ v srednjeevropskem okviru na začetku \\ 21. stoletja
}

\section{ZUSAMMENFASSUNG}

In Zusammenhang mit der EU-Osterweiterung aktualisieren sich in den Ländern, die zu der existierenden Gemeinschaft neu beitreten, nicht nur Fragen der nationalen Interessen, sondern auch nationaler Identität, einschließlich kultureller Identität. Die Schlüsselfrage heißt: außerder wirtschaftlichen Ebene gibt es in der Weltder Kunst und des Denkens etwas Wertvolleres, was unser Beitrag zur neuen erweiterten Gemeinschaft sein könnte und was die existierende EU-Welt bereichern könnte?

Von dieser Perspektive ist auch das musikalische Kulturerbe neu zu durchdenken - worin liegt denn eigentlich die tschechische Musikidentität und was könnte man im europäischen Kontext als spezifisch tschechisch bezeichnen? Auf dem Gebiet der Kunstmusik sind diese Aspekte am deutlichsten in der Musik des 19. und 20. Jahrhunderts zu definieren, und die liegen eher

1) in deutlich gepflegter nationalen Spezifität der absichtlich folkloregebundenen Musiksprache und 2) in der aus böhmischer Geschichte und Mythologie abgeleiteten Thematik, einschließlich der Benut-
POVZETEK

$\mathrm{V}$ povezavi s širitvijo Evropske unije na vzhod se v državah, ki na novo vstopajo k obstoječi skupnosti porajajo ne le vprašanja o nacionalnih interesih, temveč tudi vprašanja o nacionalni in kulturni identiteti. Ključno vprašanje se glasi: ali poleg gospodarske ravni $v$ svetu umetnosti in mišljenja obstaja nekaj dragocenega, kar bi lahko bil naš prispevek novi razširjeni skupnosti in kar bi lahko obogatilo obstoječo Evropsko unijo?

Iz te perspektive izhaja premislek o glasbeno kulturni dediščini - kako se pravzaprav kaže češka glasbena identiteta in kaj bi lahko v evropskem kontekstu označili kot specifično češko?

Na področju umetne glasbe so ti vidiki najrazločneje določeni v glasbi 19. in 20. stoletja in sicer:

1) v skrbno negovani nacionalni specifiki s folkloro povezanega glasbenega jezika in

2) v tematiki, ki se opira na češko zgodovino in mitologijo, vključno $\mathrm{z}$ uporabo dveh najstarejših verskih pesmi ("Hospodine, pomiluj ny", "Svatý Václave") kot simbolov tisočletne nacionalne zgodovine ter husitskih pesmi ("Kdož jste boží bojovníci" idr.) kot simbolov upora in bojevniškega duha. 
zung der zwei ältesten religiösen Lieder ("Hospodine, pomiluj ny", "Svatý Václave") als Symbole der tausendjährigen nationalen Geschichte und den Hussitenlieder (wie "Kdọ̌ jste boží bojovníci» u.a.) als Symbole des Widerstands und des Kampfgeistes. Die Häufigkeit, mit der diese Elemente vertreten sind, ist während der letzten cca 150 Jahre deutlich im Zusammenhang mit der Entwicklung der historischen Lage zu verfolgen: besonders in den Zeiten des Kampfes um entsprechende politische Vertretung (1848-1918) und der Bedrohung der nationalen Existenz (Kriegsjahre, Zeiten der militärischen Besatzung und totalitären Regime) wächst diese Häufigkeit der erwähnten Elemente. Die neu gesehene Integrierung der ältesten Musikdenkmäler mit religiösen Konnotationen kann man sogar bis zu den Werken jüngster Generation tschechischen Komponisten folgen - ein Beweis, dass diese Problematik auch heute noch als aktuell empfunden wird.
Pogostost s katero so ti elementi zastopani, je med zadnjimi cca. 150 leti mogoče zaslediti v povezavi $\mathrm{z}$ razvojem zgodovinskega položaja: posebno v obdobju boja za ustrezno politično zastopništvo (1848-1918) in ogroženosti nacionalne eksistence (vojna leta, čas vojaške okupacije in totalitarnih režimov) narašča pogostost omenjenih elementov. Novo videno povezovanje najstarejših glasbenih spomenikov z religioznimi konotacijami je moč zaslediti celo $\mathrm{v}$ delih najmlajše generacije čeških skladateljev - dokaz, da je ta problematika še danes aktualna.

Sehr geehrte Damen und Herren,

Die Einladung, an diesem Symposium über musikalische Identität Mitteleuropas teilzunehmen, hat mich besonders interessiert, es ist ein Thema, das ich immer wieder antreffe und das die rasche Entwicklung der letzten Jahre und Monate in immer neues Licht und neue Kontexte rückt.

1) Unser Blick auf die neue Landkarte Europas mit eingezeichneter vertikaler Kette von acht neuen kontinentalen EU-Mitgliedern von Estland bis Slowenien erinnert an den etwas westlicheren "Cordon sanitaire Abkommens; diesmal aber (glücklicherweise!) mit keinen Grenzkorrekturen verbunden, sondern mit der einmaligen Gelegenheit diesen Raum zur wirtschaftlich und politisch kooperierenden westlichen Hälfte des Kontinents zu integrieren. Für die über 70 Millionen Menschen, die lange Jahrzehnte unter sowjetischer Herrschaft lebten, ist das eine einzigartige und lange erhoffte Chance wieder "zurück nach Europa», wie viele der Wahlparolen der Zeit nach der Wende hießen, zu kommen, die tragischen Folgen der Kriegs- und Nachkriegszeit zu überwinden (wie schwer es geht, konnten wir nach 13 Jahren lange erzählen!) und die Zukunft der eigenen Länder fest mit den heutigen EU-Mitgliedsstaaten zu verbinden. Die wirtschaftliche und geopolitische Dimension dieses Prozesses und dessen Vorteile für die neuen Mitglieder sind klar; was können aber dagegen die neuen Mitgliedsstaaten den heutigen EULändern außer dem guten Willen, wichtigen geopolitischem Raum, stufenweise sich erholender Wirtschaft und schwer deformierter Wertskala der mittleren Generationen überhaupt anbieten? Werden wir - mindestens in den ersten Jahren - nur zu Nettoempfängern und einem schwarzen Loch der 
EU-Finanzen, oder gibt es etwas Wertvolleres, was unser Beitrag zur neuen erweiterten Gemeinschaft sein könnte, was nicht verschwinden würde und was die existierende EU-Welt bereichern könnte?

2) Kurz nach der Wende haben wir gehofft, dass z.B. unsere Erfahrung mit der Totalität zu solchem Beitrag werden könnte, aber sobald solche SchwarzWeiß-Betrachtung mehr Farben und Konturen bekam, haben wir festgestellt, dass auch viele EU-Länder eigene schattenhafte geschichtliche Perioden hatten, die sie mühsam verarbeiten, und dass jede Erfahrung nur schwer übertragbar ist - mit dem neuen Umdenken der eigenen Identität bleibt schließlich jeder allein.

Die Wunde nach dem Eisernen Vorhang vernarbt mühsam, aber deutlich und die positiv ausgeklungenen Volksabstimmungen in den »neuen Ländern" haben auch den mehrheitlichen Willen der Bürger Mittelosteuropas zur Integration gezeigt. Es bleiben aber in dem "neuen Raum" noch viele Ressentiments und lokale Spannungen, die in den kommenden Jahrzehnten beseitigt werden müssen. Die neuen, von den geschichtlichen Problemen unbelasteten und global denkenden Generationen (wenn sie nicht einer ahistorischen Apathie verfallen) sind die Hoffnung in dieser Hinsicht. Die Politiker dagegen schaffen neben vielem Positiven auch viele kleine Konflikte zu produzieren - besonders die heißen Vorwahlmonate ${ }^{1}$ erwecken in verschiedenen Ländern wiederholt populistische Tendenzen und Äußerungen, die dann in ruhigeren Zeiten Diplomaten mit Mühe relativieren.

3) Gibt es also etwas wie eine mitteleuropäische Identität, und wenn ja, was ist das eigentlich? Viele Technokraten, die die Welt als ein gigantischer CashFlow sehen, lächeln spöttisch über solches Wort und gar über solche Überlegungen, aber jeder, der mit offenen Augen von Prag über Wien nach Ljubljana fährt, die Barockkirchen auf den Hügeln und die Statuen von Johannes Nepomuk auf den Brücken sieht, braucht keine große Mühe um das Gefühl der Zusammengehörigkeit zu spüren - mindestens die südliche Hälfte der Länderkette der neuen EU-Länder mit ihrer Jahrhunderte dauernden gemeinsamen Geschichte hat neben vielen Ressentiments auch ein geschichtlich verankertes starkes gemeinsames Lebensgefühl. Den Brahms und Dvořák, Musil, Kafka und Hašek, Mahler und Janáček, Plečnik, Klimt und Mucha verstehen die Mitteleuropäer gut. Die Kultur, der Lebensstil, die Wertskala, die gemeinsame

1 Einer der führenden tschechischen Politiker konnte zum Exemplum dienen - seine Einstellung zur europäischen Integration besteht u.a. aus zwei Grundideen, und zwar A) dass Tschechien eigentlich keinen speziellen Beitrag für das integrierte Europa leisten kann, und B) dass uns unsere spezifische tschechische Identität so am Herzen liegen sollte, dass wir eigentlich die Integration lieber meiden sollten, um die wertvolle Identität zu bewahren; seine politische Partei will uns aber jedenfalls mit allen Kräften dabei gegen jeden ausländischen Druck helfen. Das Problem liegt daran, dass die Aussage A) an die EU gerichtet wurde, die B) dagegen an das einheimischen Publikum, besonders an den Wahlkundgebungen. Seine Lieblingswarnung, dass unser Land in der EU als ein Zucker im Kaffee zergeht, sagt viel; die Fragen, wieso uns dabei unsere wertvolle und verteidigungswerte Identität nicht rettet und wieweit es eigentlich Schade ist, wenn wir da sowieso keinen Beitrag leisten können, bleiben freilich unbeantwortet. 
Geschichte und (was viele in unserem meist atheistischen Land Europas ungern hören) die religiösen Wurzeln - das alles ist mindestens ein Kleinstnenner auf den wir uns bestimmt einigen können, auch wenn die Entwicklung der zweiten Hälfte des letzten Jahrhunderts zwischen den zwei Hälften dieses Raumes eine tiefe politische und wirtschaftliche Kluft (noch dazu geographisch unlogisch und meanderhaft gezogene ${ }^{2}$ ) geschmiedet hat.

4) Wie wäre es dann mit der tschechischen Identität? Ich lasse die psychologische Eigenschaften, wie das tschechische Humor, und die spezifischen Details des lokalen politischen und gesellschaftlichen Lebens beiseite und komme gleich (endlich) zum Bereich Musik. Hier könnte man sagen, dass mindestens die tschechische Musik des 19. Jahrhunderts ihren eindeutig charakteristischen Beitrag zum Panorama der europäischen Musik geleistet hat, und zwar mindestens in zwei Aspekten:

- in deutlich gepflegter nationalen Spezifität der absichtlich folkloregebundenen Musiksprache und

- in der aus böhmischer Geschichte und Mythologie abgeleiteten Thematik, einschließlich der Benutzung der zwei ältesten religiösen Lieder (»Hospodine, pomiluj ny«, "Svatý Václave«) als Symbole der tausendjährigen nationalen Geschichte und den Hussitenlieder (wie »Kdož jste boží bojovníci» u.a.) als Symbole des Widerstands und Kampfgeistes. Die Verkaufte Braut, Böhmische Tänze, Dvořák der mittleren Periode ${ }^{3}$ oder Janáček der Vorkriegsjahre könnten als Beispiel für das erste, Mein Vaterland, Libuše oder Werke jüngeren Dvořáks ${ }^{4}$ für das andere als elementare Beispiele dienen.

Die kritische Fragestellung der postmodernen Zeit bezweifelt aber auch das - so hält z.B. der amerikanische Musikwissenschaftler Michael Beckerman sogar die erste Prämisse für überwunden und unbeweisbar und argumentiert dabei mit der Tatsache, dass man die Einzelelemente, die in der zweiten Hälfte des 19. Jahrhunderts für typisch tschechisch gehalten und in dem Sinne auch verwendet wurden (wie der Polkarhythmus, bestimmte melodische und harmonische Elemente usw.), auch in anderen Werken nichtböhmischer Komponisten der Zeit finden kann und dass wir also keinesfalls von einer spezifischen tschechischen Musiksprache der Zeit sprechen können - die gäbe es einfach nicht ${ }^{5}$.

Diese Einstellung ist völlig im Geiste der postmodernen kritischen Skepsis zu verstehen, die die nationalistisch klingende Argumentation eliminiert und

2 Dazu eine heute unglaublich klingende, aber trotzdem wahre Nachkriegsgeschichte - ein Wiener Unternehmer tschechischer Herkunft war im Frühling 1946 schon verunsichert wegen der immer wieder in der Wiener Stadtmitte kursierenden russischen Soldaten, während in Prag keine Russen, eine Koalitionsregierung und an der Prager Burg Präsident Beneš waren. Er hat also sein Vermögen auf zwei Teile geteilt; einen Teil in den Wiener und den anderen in den Prager Banken deponiert. Welche Hälfte er schließlich gerettet hat, ist uns heute klar, aber unter dem damaligen Zeitpunkt betrachtet war das eine legitime Überlegung!

3 Mährische Gesänge, Slawische Tänze und Rhapsodien usw.

4 Husitská, Můj domov, Kantate Dědicové Bílé Hory (Die Erben des Weißen Berges) usw.

5 Siehe Michael Beckerman: In Search of Czechness in Music, $19^{\text {th }}$ Century Music 10, No.1 (1986), S. 61-73. 
die Problematik eher sachlich und materiell-technisch betrachtet, aber ist auch im Sinne der Tendenz, durch scharf formulierte Stellungnahmen eine Diskussion zu provozieren. Es ist auch logisch, dass es von außen, speziell von der emotionell an mitteleuropäischen Konflikten unbeteiligten Übersee kommt, denn bei den historischen Themen hat diese kritische Distanz besonders der angelsächsischen Historiker und Musikhistoriker viel Wertvolles zur Überwindung der nationalistisch belasteten Standpunkte des 19. und der ersten Hälfte des 20. Jahrhunderts beigetragen. Übrigens ich meine, dass die tschechische Gesellschaft in ihrer wachsenden kritischen Distanz zum Nationalismus des 19. Jahrhunderts (auch dank der Diskussionen der agileren Historiker) schon so weit ist, dass ein tschechischer Musikwissenschaftler, der die gleiche Meinung heutzutage äußern würde, nicht für einen Nestschänder gehalten würde und dass dann eine sachlich kritische Diskussion folgen könnte.

$\mathrm{Zu}$ dieser ist aber bisher kaum gekommen. Ich sehe dafür zwei Gründe. Erstens: die Auseinandersetzung mit der Welle neuer Informationen, Strömungen und Tendenzen nach der Wende ist so anspruchsvoll, dass eine Diskussion in der tschechischen musikalischen Öffentlichkeit über solche Themen noch nicht auf die Tagesordnung kam (aber bestimmt künftig kommt und ich freue mich recht darauf!). Der zweite Grund, warum eine so extrem negative Einstellung nicht von einem Insider kommt, sehe ich aber in der notwendigen Kenntnis der musikalischen Kontexte - was einem Tschechen tschechisch klingt oder gar vor 130 Jahren tschechisch klang, kann man auch bei extensiver Kenntnis der Musik der Zeit schwer von außen beurteilen ohne den ganzen Kontext zu erleben, zu erfühlen und zu durchdenken. Ein praktisches Gegenargument: wenn das Material der tschechischen Musik der zweiten Hälfte des 19. Jahrhunderts wirklich so universal und ohne jenen nationalen Akzent wäre (und die damaligen Hörer es auch so empfunden hätten), warum hat dann der gute Geschäftsmann Simrock den jungen Dvořák so energisch beauftragt, immer weitere böhmisch und slawisch klingende Stücke, die sich so gut verkaufen, zu schreiben? Und wie sollte es der arme Dvořák dann bei Nichtexistenz einer die böhmische und slawische Welt definierenden Musiksprache eigentlich machen, um die gewünschten Stücke entsprechend klingen zu lassen?

5) Im Laufe des 20. Jahrhunderts haben die beiden Wirkungskräfte (die Benutzung der folkloregebundenen Elemente und der patriotischen Thematik) in der tschechischen Musik eine komplizierte Entwicklung erlebt. Die im dritten Viertel des 19. Jahrhunderts geborene Generation (L. Janáček *1854, V. Novák *1870) hat vor der Jahrhundertwende und in den Vorkriegsjahren aus den intensiven Folklorestudien vieles im eigenem Kunstschaffen benutzt, wobei ihr Interesse mehr der modalen, primär vokalen, emotionelleren und im Ausdruck reicheren mährischen und slowakischen Volksmusik galt ${ }^{6}$, als der

\footnotetext{
${ }^{6}$ Leoš Janáček: Lachische Tänze, Rákós Rákoczy, die Rekrutenszene und die Hochzeitszene in Jenůfa und viel anderes., Vítězslav Novák: Slovenské spevy (Slowakische Gesänge), Slovácká svita (Slowakische Suite), Lieder, Chöre u.a.
} 
mehr instrumentalen und eher regelmäßig periodischen böhmischen. Die Jahre des ersten Weltkriegs setzen erneut noch Akzent auf patriotische Themen ${ }^{7}$, in den frohen und sachlichen Zwanziger Jahren verschwand dagegen die Folkloristik sowie der Patriotismus fast völlig ${ }^{8}$ - die Pariser Six, Strawinski, Jazz und Zivilismus waren die starken Inspirationen der tschechischen Musik der Zwischenkriegszeit. Paradoxerweise tauchte das Interesse an der künstlichen Bearbeitung der böhmischen Volkmusik am Anfang der 30er Jahre in Paris, einer Bastion der erwähnten innovativen Strömungen, wieder auf - B. Martinů (*1890), der dort damals schon fast ein Jahrzehnt lebte und alle erwähnten Impulse erprobte, hat sich in einer Reihe seiner Bühnen- und Konzertwerke ${ }^{9}$ erneut durch böhmische Volksmusik inspirieren lassen, wobei er aber schon mit den Augen sah, die Petruschka, Les Noces und Histoire d'un soldat gut kannten. In den späten 30er Jahren, in der Zeit der wachsenden Gefahr und Bedrohung, erschienen die beiden genannten Tendenzen auch bei anderen Autoren wieder; einschließlich V. Novák ${ }^{10}$ und der besonders bedrohten Komponisten jüdischer Herkunft (E. F. Burian, P. Haas, V. Ulmann u.a.).

In den Kriegsjahren desto mehr - viele der böhmischen und mährischen Komponisten einschließlich der im ganz verschiedenen Klima der Zeit zwischen den Kriegen geformten jüngeren Generation, reagierten auf die akute Bedrohung mit der Zuneigung zur patriotischen Thematik ${ }^{11}$ und zu den Elementen der Volksmusik des Landes ${ }^{12}$.

Ein weiteres Paradox - diese Tendenzen, die in den kritischen Kriegsjahren ein Akt der aktiven Selbstverteidigung und Identitätsschutz waren, wurden bald danach, in den Zeiten des harten stalinistischen kommunistischen Regimes (in schlimmster Form 1948 - cca 1954) zur durch Machtdruck erpressten Orientierung - die ästhetischen Prinzipien des schdanowistischen "sozialistischen Realismus" verlangten beides: ideologische Thematik (natürlich ohne den religiösen Aspekt und in den eroberten Ländern des Ostblocks lieber »internationalistische" als patriotische) sowie die tonale und folkloristisch gefärbte $\mathrm{Mu}-$ siksprache. Das zweite, intelligent und nichtideologisch gemacht, wurde für viele Komponisten (einschließlich des mittleren Lutoslawski und jüngeren Ligeti!) zum ehrenvollen und akzeptablen Kompromiss, alsbald dann die politische Lage im Ostblock etwas lockerer wurde (d.h. seit den späteren 50er Jahren), verschwand diese Tendenz entsprechend dem schwächenden politischen

7 Josef Suk: Meditation über dem alttschechischen Choral zum hl. Wenzel, was das Böhmische Streichquartett, wo Suk zweite Violine spielte, auf seinen Konzerten in den Kriegsjahren am Anfang statt der verordneten österreichischen Staatshymne spielte, u.a.

8 Bis auf bestimmte Ausnahmen, wie die Kantaten von L. Vycpálek u.a.

9 Bohuslav Martinů: Špalíček, Borová, Kytice (Blumenstrauß), später Veselohra na mostě (Komödie auf der Brücke) und viele anderen Werke.

${ }^{10}$ Jihočeská svita (Südböhmische Suite) u.a.

${ }^{11}$ V. Novák: Svatováclavský triptych (Triptych des hl.Wenzel), Májová symfonie (Maisymphonie), Lieder und Chöre.

${ }^{12}$ Z.B. M. Kabeláč, K. Slavický, J. Kapr, aber auch die vor dem Krieg meistens international orientierten »Theresienstädter Autoren«, wie P. Haas oder G. Klein. 
MUZIKOLOŠKI ZBORNIK • MUSICOLOGICAL ANNUAL XL

Druck und wurde in den dynamischen 60er Jahren mit ihrem schnellen Nachholbedarf an Verarbeitung westeuropäischer Nachkriegsimpulse völlig »out«. Der Serialismus, Aleatorik, Timbremusik, Elemente Neuer Musik, das alles war sehr weit von jener lokalen oder gar nationalen Färbung und floss in den Hauptstrom der europäischen Schaffensaktivität, die mit ihrem universalen Musikmaterial minimale nationale Spezifität enthielt. Eine echt überraschende Wende kam in den 70er Jahren mit der Neoromantik und später Polystilistik, wo im Rahmen dieser Tendenz neu gesehene Elemente der Volksmusik wieder aktuell wurden, diese Tendenz fand aber mehr Resonanz in der polnischen ${ }^{13}$ als in der tschechischen Musik ${ }^{14}$. In Böhmen provozierten dagegen die militärische Unterdrückung des "Prager Frühlings 1968« und die folgenden repressiven Jahre der »Normalisierung « bei den Komponisten einen starken künstlerischen Protest: Miloslav Kabeláč (E fontibus bohemicis, Proměny chorálu [Die Metamorphosen des Chorals] I und II), Petr Eben (Vox clamantis und religiös inspirierten Werke ${ }^{15}$ ) und der in den USA lebende Karel Husa (Music for Prague 1968) sind als die bedeutendsten Beispiele zu nennen - die ältesten tschechischen religiösen Lieder und Hussitenchoräle erklangen in dieser "Stunde der Wahrheit« erneut, diesmal nicht umgegeben von romantisch chromatischer Musik, sondern von seriell (Husa) oder modal gegründeter Musiksprache.

Die folgende Welle der Minimalismus den 80er und 90er Jahre bot weltweit der Verarbeitung von Folkloreelementen wieder neue technische Grundlage, sowie die elektroakustische Musik, die, gleich weltweit, eine unübersehbare Tendenz zur Arbeit mit den Klangelementen besonders der außereuropäischen Musikkulturen enthält. In der Stilpluralität der letzten Jahrzehnte bildet aber diese Tendenz in der Musik der mittellosteuropäischen Länder eher eine Minorität, obwohl dieser Trend bis zur heutigen jungen Komponistengeneration reicht.

Die übrige in diesem Raum in den letzten Jahrzehnten Komponierte Kunstmusik, die keine ausgesprochene oder angedeutete Folkloreelemente enthält, besonders wenn sie auf den rationellen kompositorischen Techniken basiert, ist meistens kaum der Landesherkunft ihres Verfassers beizuordnen; die für heutige Zeit typische weltweite Migration der Autoren verstärkt natürlich diese überwiegende Universalität der Musiksprache, die einerseits stilistisch extrem differenziert ist, aber gleichzeitig zur Megaintegration der historischen, ethnischen und artifiziellen sowie nonartifiziellen Musik stark tendiert. Meistens nur eine Art der Klangvorstellung oder Sensitivität zum Musik- und Klangmaterial kann heutzutage auf die Verankerung des Komponisten in einer bestimmten musikalischen Landeskultur deuten.

\footnotetext{
${ }^{13}$ In den 70er Jahren Orchesterwerke wie z.B. Krzesany vom polnischen Komponisten W. Kilar u.a.

${ }^{14} \mathrm{Da}$ sind die Werke von Z. Lukáš, J. Krček u.a. aus dieser Zeit zu nennen.

${ }^{15}$ Wobei im Schaffen von Petr Eben die Elemente historischer Musik einschließlich der religiösen eine starke Rolle vom Anfang der 50er Jahre bis heute spielten, in dem Sinne war er eine Ausnahme, die die Änderungen des Zeitgeistes nicht kopierte.
} 
MUZIKOLOŠKI ZBORNIK • MUSICOLOGICAL ANNUAL XL

6) Worin liegt also die tschechische und mitteleuropäische Musikidentität heute? Wenn ich eine Antwort auf diese extrem schwierige Frage versuchen darf, dann:

a) Bei der neu geschriebenen artifiziellen Musik materiell genommen in gelegentlicher Verarbeitung des lokalen Folklorematerials oder in absichtlichen Anspielungen aufdie Musikgeschichte der Region. Und der historische Überblick zeigte uns, dass die Intensität, mit welcher die tschechischen Komponisten von Mitte des 19. Jahrhunderts bis heute "ad fontes« - zu diesen zwei Quellen gegriffen haben, sich sehr deutlich mit den dynamischen und schwierigen historischen Zeiten deckte: mit dem Kampf der expandierenden tschechischen Gesellschaft für mehr Raum in der K.u.k.-Monarchie (Smetana, Dvořák, Janáček, Novák), mit der Zeit des ersten Weltkriegs (Suk, Janáček...), dann wieder des zweiten Weltkriegs (Novák, Kabeláč, Kapr, K. Slavický ...), erzwungen in stalinistischen Zeiten und schließlich als Protest während der Krise nach 1968 (Husa, Kabeláč, Eben ...). Aber auch in dem Schaffen der heutigen jüngsten Generation tschechischer Komponisten sind die beiden Aspekte nicht ganz "out" die Folkloreelemente ${ }^{16}$ sind wieder $\mathrm{zu}$ finden sowie die neu gesehene Religiosität ${ }^{17}$ - hier vereinigt sich ein Nachholbedarf nach Jahrzehnten des hart atheistischen Regimes mit einer aufrichtigen Suche nach dauernden Werten; das Leben in Zeiten des wachsenden Wohlstands und allgemeiner Hoffnungen, aber zugleich in der überwiegend ideenleeren Konsumgesellschaft ist für empfindliche junge Künstler zu einer verunsichernden Herausforderung geworden.

b) Wenn wir die Musikkultur im ganzen betrachten, dann liegt die mitteleuropäische Musikidentität bestimmt auch in der starken lokalen interpretatorischen Tradition, wobei die österreichische und tschechische Musikkultur wahrscheinlich die stärkste immanente Tradition haben (siehe die Wiener Pflege der traditionellen Sonorität der Instrumente einschließlich des akustisch forschenden »Instituts für echten Wiener Klang «).

7) Was könnte dann die mitteleuropäische musikalische Identität als Beitrag des neuen Europa anbieten? Allgemein gesehen besonders eine denkwürdige Tradition des in eigener Kultur und Geschichte verankerten Schaffens und der Interpretation und ein kultiviertes Milieu mit hohem Ansehen der Musik als Beruf und als Geistesaktivität. Ist das wenig?

Ich weiß, ich habe dabei nichts besonderes entdeckt, wahrscheinlich mehr Fragen als Antworten angeboten und diese Zusammenfassung kann eher banal klingen. Aber um in einem Bogen zurück zur Anfangsfrage zu kommen bei rascher Entwicklung der europäischen Integration in den letzten Monaten erachte ich als besonders wichtig auf diese starken immanenten Kulturwurzeln immer wieder zu verweisen. Vor allem in den postkommunistischen

\footnotetext{
${ }^{16}$ Besonders bei den jungen mährischen Autoren (L. Foltýnová u.a.).

${ }^{17}$ M. Rataj, M. Ivanovič, T. Pospíšil, J. Koželuhová, R. Hejnar und viele anderen.
} 
MUZIKOLOŠKI ZBORNIK • MUSICOLOGICAL ANNUAL XL

Ländern, wo der im neuen Anzug wieder frontal siegende Materialismus die Geisteswerte wieder in die Ecke rasant wegschiebt, muss man über die Kulturidentität als den wichtigsten Beitrag zur europäischen Polyphonie immer wieder sprechen und gleichzeitig die notwendige Sensitivität gegenüber den totalitären und nationalistischen Tendenzen pflegen. Wie der im März 1948 (nur einige Tage nach der kommunistischen Machtergreifung unter mysteriösen Umständen) verstorbene tschechoslowakische Außenminister Jan Masaryk, der Sohn des Gründers und ersten Staatspräsidenten T. G. Masaryk, sagte im Londoner Kriegsasyl seinem Sekretär Viktor Fischl (dem späteren israelischen Diplomaten Avigdor Dagan): „Der Mensch muss immer nach oben schauen. Und die Kunst ist hier um ihm dabei zu helfen."

Ich bedanke mich von Herzen für dieses aktuelle Thema der Konferenz und für ihre Einladung. 